\title{
The Effect of Stress, Worries and Resilience on Sleep Quality of Chinese Population During the Normalization of COVID-19 Pandemic Prevention and Control
}

\section{Hongfei Ma}

China Medical University

Minghui Liu

China Medical University

Shuang Zhao

China Medical University

Meijun Long

China Medical University

\section{Yucong Diao}

China Medical University

\section{Ziyi Feng}

China Medical University

Hui Wu

China Medical University

Yang Wang ( $\nabla$ yangwang@cmu.edu.cn )

China Medical University https://orcid.org/0000-0002-9113-157X

\section{Research}

Keywords: COVID-19, pandemic, sleep quality, stress, worries, resilience

Posted Date: November 10th, 2021

DOI: https://doi.org/10.21203/rs.3.rs-1015671/v1

License: (a) This work is licensed under a Creative Commons Attribution 4.0 International License. Read Full License 


\section{Abstract}

Background: The COVID-19 pandemic has spread worldwide and has attracted considerable attention. This study aims to investigate the sleep quality of the public during the normalization of COVID-19 pandemic prevention and control in China, as well as related factors that affect sleep quality.

Methods: A web-based cross-sectional survey was implemented from Feb $2^{\text {nd }}$ to Feb $9^{\text {th }}, 2021$ in China. A total of 613 subjects from 27 provinces and 4 municipalities participated in this study. Questionnaire including Pittsburgh Sleep Quality Index (PSQI), COVID Stress Scale (CSS), COVID-19 Worry Scale and 14item Resilience Scale (the RS-14) as well as demographic characteristics and pandemic-related information was used to measure sleep quality, stress, worries and resilience of Chinese population. Hierarchical multiple regression analysis was conducted to explore the associated factors of sleep quality.

Results: The prevalence of poor sleep quality among 613 participants was $41.1 \%$ (95\% $\mathrm{Cl}=37.2 \%-45 \%)$. Regression analysis indicated that chronic diseases, medical visits, occupation, and negative life events were factors affecting sleep quality. Stress and worries were positively associated with score of sleep quality, while resilience was negatively associated with score of sleep quality.

Conclusions: During the normalization of COVID-19 pandemic prevention and control, Chinese public has poor sleep quality. Effective interventions should be taken to decrease people's COVID-19 stress and worries. Resilience enhancement is also an important factor to be considered to improve people's sleep quality.

\section{Background}

At the beginning of 2020, the outbreak of respiratory diseases caused by the severe acute respiratory syndrome coronavirus 2 (SARS-CoV-2) has emerged around the world, which is highly infectious and pathogenic [1]. On 11 February 2020, WHO named the disease caused by coronavirus 2 as COVID-19 [2]. So far, the infection of COVID-19 is still spreading, and this virus poses a serious threat to public health. The COVID-19 has also caused huge damage in China. As of 7 April 2021, the COVID-19 pandemic in China has resulted in 102,970 infections, of which 4851 have died [3]. Since the COVID-19 outbreak, it has triggered a global recession, while social distance, self-isolation and travel restrictions have reduced the workforce and led to loss of many jobs [4]. At the same time, the pandemic also changed people's lifestyles which may include eating habits, exercise habits, learning and working style, et al. Due to the impact of the pandemic of COVID-19, psychological pressure of individuals was increased, sleep quality decreased, anxiety, depression, loneliness and a series of mental health problems appeared [5]. A population survey in China showed that during the COVID-19 pandemic, individuals were widely stressed, with $52.1 \%$ of 263 participants feeling fear or anxiety about the pandemic [6]. Moreover, Liu and Xie's survey on the psychological status of the Chinese population showed that $27 \%$ of the respondents were depressed, and $33.2 \%$ of the respondents suffered from poor sleep quality $[7,8]$. 
Sleep, as one of the most important human behaviors, is highly sensitive in life and susceptible to various factors [9]. The negative impact of the COVID-19 pandemic on economy, education, work and life can increase the psychological stress of individuals, which can further affect the quality of sleep [10]. In a study of 365 COVID-19 patients surveyed, $69.6 \%$ reported having at least one sleep disturbance during the COVID-19 pandemic, with frequent awakenings being the most common problem [11]. Similarly, a survey on sleep quality among 1,931 front-line medical workers showed that the prevalence of sleep disorders was $18.4 \%$ [12]. However, decreased quality of sleep appeared not only in patients and high-risk occupations, but also in ordinary population. Stanton's survey of the Australian public showed that $40.7 \%$ of respondents said their sleep quality had declined due to the outbreak of COVID-19 [13]. Meanwhile, the general population in China also suffered from poor sleep quality at the beginning of the COVID-19 outbreak. Ji's research showed that $22.4 \%$ of respondents in China reported poor sleep quality during the early stage of COVID-19 pandemic [14]. Nowadays, the domestic pandemic of COVID-19 has been under control with the efforts of Chinese government, but the psychological impacts may still persist [15]. Therefore, during the normalization of COVID-19 pandemic prevention and control, the sleep quality of the Chinese public and its influencing factors are worthy of study.

There was widespread stress among individuals due to the impact of the COVID-19 pandemic. Some scholars divided this stress into five areas, including danger and contamination fears, fears about socioeconomic consequences, xenophobia, compulsive checking and reassurance seeking, and traumatic stress symptoms about COVID-19 [16]. First of all, COVID-19 viruses are associated with severe illness or death and are highly infectious, so many people exhibit fears of contacting with possibly contaminated objects or patients [17]. Secondly, the outbreak of COVID-19 also had a serious impact on global economy. For example, people's incomes would be reduced, unemployment would rise, and transportation or services would be limited, which would increase the stress of individuals [4]. Thirdly, at present, Chinese pandemic prevention and control was relatively stable, but the existence of imported cases and the safety problems of imported food will also cause stress on domestic public $[18,19]$. Lastly, many people exhibit compulsive checking and reassurance seeking regarding possible pandemic-related threats or experience traumatic stress symptoms, such as frequent nightmares and intrusive thinking $[16,20-$ 22]. People's exposure to stress is associated with decreased sleep quality, while people with higher perceived stress tend to have lower sleep quality. A survey of 1,262 Wuhan residents who were experiencing great stress as a result of the COVID-19 pandemic, $30.6 \%$ of the residents showed sleep problems [23].

Worries have common characteristics of repetitive cognitive mechanism, namely negative emotions and orientation away from the present [24]. In public health emergencies, individuals generally have worries about the future development of the events, as well as worries about their own study, work and life [25]. From the beginning of 2020 to the present, the COVID-19 pandemic has lasted for more than a year. Affected by the long-lasting COVID-19 pandemic, individuals worry about the uncertainty of future economic income, employment environment, and future life [26]. Previous studies have found that during the COVID-19 pandemic, people's worries caused a decline in sleep quality and an increase in the 
frequency of insomnia [27]. Similarly, The study of 584 subjects in Argentina found that the incidence of insomnia during the COVID-19 pandemic was $45 \%$, which has been linked to daytime worries [28].

Resilience is defined as "having not only important psychological skills or abilities, but also the ability of the individuals to use family, social and external support systems to better cope with stress" [29].

Previous research has found that an individual's resilience is associated with their sleep quality, and low resilience leads to poorer sleep quality [30]. But Some individuals who are stressed and worried don't tend to suffer from poor sleep quality, and part of the reason was the high resilience. Because they were able to overcome difficulties in adversity and adapt to a new situation with a positive attitude and manner, even if the beginning of an event is negative [31]. During the COVID-19 pandemic, the impact of resilience on sleep quality is particularly obvious. There were some front-line medical staff who had better sleep quality because they were determined to overcome COVID-19, had firm willpower, and were well-adapted to the current bad life [32]. On the contrary, during the COVID-19 pandemic, there were still some ordinary people with poor resilience and their sleep quality was affected. Ephraim believed that when facing the COVID-19 pandemic, people with lower resilience lacked the ability to seek help and social support, and were more likely to feel lonely and helpless, which further affected their sleep quality [27].

The purpose of this study was to investigate the sleep quality of individuals in China during the COVID-19 pandemic through a cross-sectional study. To explore the influencing factors of sleep quality, particularly the effects of stress, worries and resilience experienced during the COVID-19 pandemic on sleep quality. It provided a theoretical basis for the study of sleep quality in the pandemic of COVID-19. Most importantly, during the normalization of COVID-19 pandemic prevention and control, there has been little research on individual's sleep quality and its influencing factors. Therefore, this study has theoretical and practical significance.

\section{Methods}

\subsection{Participants and Procedure}

A web-based cross-sectional survey was implemented from Feb 2nd to Feb 9th, 2021 by using the Questionnaire Star platform and broadcasted through the mainstream social-media WeChat in Chinese population. A total of 618 subjects from 27 provinces and 4 municipalities participated in this study. Participants in this study met the following inclusion criteria: age $\geq 18$ years; be able to read Chinese and use the Internet and WeChat to complete the questionnaire independently; willing to participate and provide signed web-based informed consent. This study has been ethically approved by the Human experiment Committee of China Medical University, and all the research processes are in line with ethical standards. Each participant participated in the survey voluntarily and signed an informed consent form online before the investigation. Of the 618 participants, 5 subjects were excluded from the final analysis due to too short answer time ( $<3$ minutes). The final sample size was 613 , with the effective response rate was $99.19 \%$.

\subsection{Measures}




\subsubsection{Demographic characteristics and pandemic-related information of the participants}

The demographic characteristics of the participants included gender (male, female), age ( $<30$ years, $\geq 30$ years), marital status (unmarried, other), Chronic diseases (yes, no), medical visits (0-2 times, $\geq 3$ times), occupation, education and annual income. Occupation was categorized as students, employed personnel and temporary unemployed / retired personnel. Education was categorized as below high school diploma, junior college / bachelor's degree, Master degree and above. Annual income (RMB) was classified as $\leq ¥ 20,000$ ( $\leq$ US $\$ 3,052.04$ ), $¥ 20,001$ to $¥ 50,000$ (US $\$ 3,052.04$ to $\$ 7,630.1$ ), $¥ 50,001$ to $¥ 100,000$ (US $\$ 7,630.1$ to $\$ 15,260.2$ ), $¥ 100,001$ to $¥ 200,000$ (US $\$ 15,260.2$ to $\$ 30,520.4$ ) and $>¥ 200,000$ (>US $\$ 30,520.4)$. Pandemic-related information included affected areas (yes, no), negative life events (yes, no), impact of the pandemic on social activities (yes, no).

\subsubsection{Measurement of sleep quality}

In this study, we used four items from the Pittsburgh Sleep Quality Index (PSQI) to assess the sleep quality of subjects over a month. The four items are a) Subjective sleep quality by asking "how good is your sleep quality?" b) Sleep duration by asking "what is your actual sleep time recently?" within 1-month lasted. c) Unable to fall asleep within 30 minutes by asking "do you have difficulty in starting sleep?" d) Easily waking by asking "do you have easy waking during sleep and early waking in the morning?" Each item had 4 options. Four options for subjective sleep quality are "very good", "good", "bad", and "very bad". Four options for sleep duration are " $\leq 5 h$ ", “5-6h", "6-7h" and " $\geq 7 h$ ". Four options for unable to fall asleep within $30 \mathrm{~min}$ and easily waking during the night or too early in the morning are "none", "less than once a week", " 1 to 2 times per week", " 3 or more times a week". Each component is scored in the range of $0-3$, and the sum of the scores of the 4 components gives the total scale score, and the total score varies in the range of $0-12$. Based on previous studies in China (cut-off value $=8$, the total scores $=21$ ), the cut-off value of poor sleep quality in this study was 4.57 (the total scores $=12$ ) by using the proportional calculation method $[33,34]$. The higher the score, the worse the sleep quality. Cronbach's a coefficient of the global score was 0.78 .

\subsubsection{Measurement of Stress}

The COVID Stress Scale (CSS) was invented by Taylor in 2020, and this scale has been translated into languages of other countries to measure individual stress related to COVID-19 [16]. The Chinese version of Covid Stress Scale is already available on the website and it include 36 items in five dimensions. The five dimensions include (1) danger and contamination fears, (2) fears about economic consequences, (3) xenophobia, (4) compulsive checking and reassurance seeking, and (5) traumatic stress symptoms about COVID-19. Items in danger and contamination fears, fears about economic consequences, and xenophobia were rated on a five-point Likert scale (1 (none) to 5 (extreme)), and respondents were asked to indicate the levels of COVID-19-related stress they had experienced in the past week. Items in compulsive checking and reassurance seeking, and traumatic stress symptoms about COVID-19 were rated on a 5 -point Likert scale ranging from 1 (never) to 5 (almost always). For these scales, respondents 
were asked to indicate how often they had compulsive check-ups or reassuring behaviors, as well as how often they had experienced problems related to traumatic stress in the past week. Summarize the scores of five dimensions, with higher scores indicating higher levels of stress associated with COVID-19. Exploratory factor analysis showed that the cumulative factor load of the scale was $78.59 \%$, and the average factor load for each dimension was $>0.5$. Cronbach's a coefficient of the global score was 0.97 .

\subsubsection{Measurement of worries}

Worries were measured by a self-compiled questionnaire which includes 6 items to measure worries of long-term impact of COVID-19 from six aspects: global economy, personal incomes, personal job opportunities, personal study and employment, personal marriage relationship and personal medical treatment. Each item is answered using a 5-point Likert scale ranging from 1 (never) to 5 (very severe) to measure the level of worries, with total score ranging from 6-30 and higher scores indicating a higher level of worries. Exploratory factor analysis showed that the cumulative factor load of the scale was $62.93 \%$ and the Cronbach's a coefficient of the global score was 0.88 .

\subsubsection{Measurement of Resilience}

The Chinese version of the 14-item Resilience Scale (the RS-14) was used to measure the resilience of the population. In previous studies on resilience, the RS-14 Chinese version has been proven to be a reliable and valid tool [35]. The scale is mainly composed of 14 items, each item is answered using a 7-point Likert scale ranging from "strongly disagree" to "strongly agree", with total scores ranging from 14 to 98 . Higher scores indicate higher levels of resilience. Cronbach's a coefficient of the global score was 0.96.

\subsection{Statistical Analysis}

All analyses were performed using SPSS version 23.0 for Windows. A two-tailed probability value $<0.05$ was considered statistically significant. First, we reported the demographic characteristics and pandemicrelated information of the subjects, and t-test and one-way variance analysis (ANOVA) were used to analyze sleep quality scores among different demographic groups and pandemic-related groups. Second, Pearson correlation analysis was performed among the four quantitative variables. Finally, multiple linear regression analysis (enter) was conducted to identify the risk factors for sleep quality, with the score of sleep quality entered as the dependent variable and potential associated variables entered as independent variables.

\section{Results}

\subsection{The demographic characteristics and pandemic-related information of participants and comparison of sleep quality score among different subgroups}

The average sleep quality score of 613 respondents was 4.08 ; of which, $252(41.1 \%, 95 \% \mathrm{Cl}=37.2 \%-45 \%)$ reported poor sleep quality (the score of sleep quality $\geq 4.57$ ). The demographic characteristics and 
pandemic-related information of participants and sleep quality score comparison among different groups is shown in Table 1. Older respondents had significantly higher sleep quality score than younger ones $(\mathrm{t}=-4.95, \mathrm{P}<0.01)$. Unmarried respondents scored lower on sleep quality than those in other conditions $(t=-4.64, P<0.01)$. Participants with lower education level had significantly higher sleep quality score $(F=2.97, P<0.05)$. Respondents with chronic diseases had significantly higher sleep quality score than people without $(\mathrm{t}=-3.68, \mathrm{P}<0.01)$. Those who had medical visits more than $\geq 3$ times in a year scored higher on sleep quality than those who had medical visits $0-2$ times $(t=-4.27, P<0.01)$. Participants who are unemployed or retired scored higher on sleep quality than students and employed personnel $(F=11.73$, $P<0.01)$. Those who had experienced negative life events scored higher on sleep quality than those who had not $(t=-4.83, P<0.01)$. Participants whose social activities were affected during the COVID-19 pandemic scored higher on sleep quality than those who were not $(t=-3.34, P<0.01)$. 
Table 1

Demographic characteristics associated with sleep quality among participants

\begin{tabular}{|c|c|c|c|c|}
\hline Variable & $N(\%)$ & $\begin{array}{l}\text { Sleep quality } \\
\text { Mean (SD) }\end{array}$ & $t / F$ & $\mathbf{p}$ \\
\hline Gender & & & -1.14 & 0.26 \\
\hline Male & 184(30.02) & $3.88(2.76)$ & & \\
\hline Female & $429(69.98)$ & $4.16(2.88)$ & & \\
\hline Age & & & -4.95 & 0.00 \\
\hline$<30$ years & 295(48.12) & $3.49(2.61)$ & & \\
\hline$\geq 30$ years & $318(51.88)$ & $4.61(2.96)$ & & \\
\hline Marital status & & & -4.64 & 0.00 \\
\hline Unmarried & $289(47.15)$ & $3.52(2.62)$ & & \\
\hline Other & $324(52.85)$ & $4.57(2.95)$ & & \\
\hline Education & & & 2.97 & 0.05 \\
\hline high school or under & $56(9.14)$ & $4.95(3.32)$ & & \\
\hline junior college/bachelor's degree & $316(51.55)$ & $4.03(2.82)$ & & \\
\hline master degree or above & $241(39.31)$ & $3.94(2.75)$ & & \\
\hline Chronic diseases & & & -3.68 & 0.00 \\
\hline No & $514(83.85)$ & $3.89(2.83)$ & & \\
\hline Yes & $99(16.15)$ & $5.03(2.77)$ & & \\
\hline Medical visits & & & -4.27 & 0.00 \\
\hline 0-2times & $515(84.01)$ & $3.86(2.78)$ & & \\
\hline$\geq 3$ times & $98(15.99)$ & $5.18(2.97)$ & & \\
\hline Annual income (RMB) & & & 1.43 & 0.22 \\
\hline$\leq 20,000$ & $57(9.31)$ & $3.96(2.99)$ & & \\
\hline $20,001 \sim 50,000$ & 105(17.13) & $4.00(2.86)$ & & \\
\hline $50,001 \sim 100,000$ & 149(24.30) & $3.81(2.97)$ & & \\
\hline $100,001 \sim 200,000$ & 164(26.75) & $3.99(2.66)$ & & \\
\hline$>200,000$ & 138(22.51) & $4.57(2.84)$ & & \\
\hline
\end{tabular}




\begin{tabular}{|lllll|}
\hline Variable & N(\%) & $\begin{array}{l}\text { Sleep quality } \\
\text { Mean (SD) }\end{array}$ & t/F & P \\
\hline Occupation & & & 11.73 & 0.00 \\
\hline students & $162(26.43)$ & $3.40(2.55)$ & & \\
\hline employed personnel & $379(61.82)$ & $4.13(2.88)$ & & \\
\hline unemployed or retired personnel & $72(11.75)$ & $5.31(2.87)$ & & \\
\hline Affected areas & & & 1.13 & 0.26 \\
\hline No & $541(88.25)$ & $4.03(2.81)$ & & \\
\hline Yes & $72(21.75)$ & $4.43(3.14)$ & & \\
\hline Negative life events & & & -4.83 & 0.00 \\
\hline No & $486(79.28)$ & $3.80(2.73)$ & & \\
\hline Yes & $127(20.72)$ & $5.14(3.03)$ & & \\
\hline Impact of the pandemic on social activities & & & -3.34 & 0.00 \\
\hline No & $70(11.42)$ & $3.01(2.75)$ & & \\
\hline Yes & $543(88.58)$ & $4.21(2.83)$ & & \\
\hline Abbreviation: SD, standard deviation & & & & \\
\hline
\end{tabular}

\subsection{Correlation between stress, resilience, worries and sleep quality}

Correlation analysis results were demonstrated in Table 2. Stress was significantly related to subjective sleep quality $(r=0.233, P<0.01)$, trouble falling asleep $(r=0.196, P<0.01)$, and easily waking at night or too early in the morning $(r=0.250, P<0.01)$. Score of worries was significantly related to subjective sleep quality $(r=0.213, P<0.01)$, trouble falling asleep $(r=0.210, P<0.01)$, and easily waking at night or too early in the morning $(r=0.177, P<0.01)$. Resilience was significantly related to subjective sleep quality $(r=-0.186$, $P<0.01)$, and trouble falling asleep $(r=-0.113, P<0.01)$. Table 2 also reported the mean (and SD) of the participants levels in stress, worries, resilience and sleep quality. 
Table 2

Correlation between stress, resilience, worry and sleep quality

\begin{tabular}{|c|c|c|c|c|c|c|c|c|}
\hline & 1 & 2 & 3 & 4 & 5 & 6 & 7 & Mean \pm SD \\
\hline 1.Stress & 1.000 & & & & & & & $72.60 \pm 24.30$ \\
\hline 2.Worry & $.544^{\star \star}$ & 1.000 & & & & & & $15.97 \pm 5.37$ \\
\hline 3.Resilience & $-.107^{\star \star}$ & -.005 & 1.000 & & & & & $66.50 \pm 13.63$ \\
\hline $\begin{array}{l}\text { 4.Subjective sleep } \\
\text { quality }\end{array}$ & $.233^{\star \star}$ & $.213^{\star \star}$ & $-.186^{* *}$ & 1.000 & & & & $4.08 \pm 2.85$ \\
\hline 5. Sleep duration & .050 & 0.17 & 0.13 & $.403^{\star *}$ & 1.000 & & & $1.18 \pm 0.83$ \\
\hline $\begin{array}{l}\text { 6.Trouble falling } \\
\text { asleep }\end{array}$ & $.196^{* \star}$ & $.210^{\star \star}$ & $-.113^{* *}$ & $.690^{\star *}$ & $.277^{\star \star}$ & 1.000 & & $0.74 \pm 0.64$ \\
\hline \multicolumn{9}{|l|}{$\begin{array}{l}\text { or too early in the } \\
\text { morning }\end{array}$} \\
\hline Abbreviation: SD, st & dard devi & tion & & & & & & \\
\hline
\end{tabular}

\subsection{Related factors of the sleep quality severity}

The results of the hierarchical regression analysis were presented in Table 3. The statistically significant variables in Table 1 were used as control variables. Stress, worries and resilience were put into the multivariate regression model as predictors. The results were shown in Table 3. Chronic diseases $(\beta=$ 0.082 , $P<0.05)$, medical visits $(\beta=0.095, P<0.05)$, occupation and negative life events $(\beta=0.102, P<0.01)$ were significantly positively associated with score of sleep quality. Students had lower score of sleep quality than unemployed or retired personnel $(\beta=-0.150, P<0.05)$, and employed personnel had lower score of sleep quality than unemployed or retired personnel $(\beta=-0.141, P<0.05)$. Stress $(\beta=0.109, P<0.05)$ and worries $(\beta=0.145, P<0.01)$ were positively associated with score of sleep quality, and resilience $(\beta=-0.107$, $P<0.01)$ were significantly negatively associated with score of sleep quality. The forest plot (Figure 1 ) also showed significant correlations with sleep quality. 
Table 3

Regression analyses with sleep quality score as the dependent variable $(n=613)$.

\begin{tabular}{|c|c|c|c|c|}
\hline \multirow{2}{*}{\multicolumn{2}{|c|}{ Variable }} & \multicolumn{3}{|c|}{ Sleep quality scores } \\
\hline & & B & $\beta$ & $\mathbf{t}$ \\
\hline \multicolumn{2}{|c|}{ Gender } & 0.409 & 0.066 & 1.738 \\
\hline \multicolumn{2}{|l|}{ Age } & 0.408 & 0.072 & 0.959 \\
\hline \multicolumn{2}{|c|}{ Marital status } & 0.457 & 0.080 & 1.082 \\
\hline \multicolumn{2}{|c|}{ Chronic diseases } & 0.631 & 0.082 & $2.023^{*}$ \\
\hline \multicolumn{2}{|c|}{ Medical visits } & 0.740 & 0.095 & $2.501^{*}$ \\
\hline \multicolumn{2}{|c|}{ Students vs. unemployed or retired personnel } & -0.970 & -0.150 & $-2.192^{*}$ \\
\hline \multicolumn{2}{|c|}{ Employed personnel vs. unemployed or retired personnel } & -0.828 & -0.141 & $-2.410^{\star}$ \\
\hline \multicolumn{2}{|c|}{ Negative life events } & 0.718 & 0.102 & $2.653^{* *}$ \\
\hline \multicolumn{2}{|c|}{ Impact of the pandemic on social Activities } & 0.653 & 0.073 & 1.913 \\
\hline \multicolumn{2}{|l|}{ Stress } & 0.013 & 0.109 & $2.405^{\star}$ \\
\hline \multicolumn{2}{|l|}{ Worry } & 0.077 & 0.145 & $3.124^{\star \star}$ \\
\hline \multicolumn{2}{|c|}{ Resilience } & -0.022 & -0.107 & $-2.800^{* \star}$ \\
\hline $\mathrm{R}^{2}$ & \multicolumn{2}{|l|}{ Adjusted $\mathrm{R}^{2}$} & $\mathrm{~F}$ & $P$ value \\
\hline 0.178 & \multicolumn{2}{|l|}{0.161} & 14.369 & $<0.001$ \\
\hline \multicolumn{5}{|c|}{$\begin{array}{l}\text { The Occupation was transformed into two dummy variables (Students vs. unemployed or retired } \\
\text { personnel, Employed personnel vs. Unemployed or retired personnel) with the unemployed or retired } \\
\text { personnel as the reference group. }\end{array}$} \\
\hline \multicolumn{5}{|c|}{${ }^{*} \mathrm{p}<0.05,{ }^{*} \mathrm{p}<0.01$ (two-tailed). } \\
\hline
\end{tabular}

\section{Discussion}

Cases of COVID-19 have increased rapidly worldwide, with human-to-human transmission accelerating significantly, and the mutated virus also showing a pandemic trend [36]. Therefore, these situations have led countries to be concerned about the impact of the COVID-19 pandemic, particularly the impact on the sleep quality of the population $[1,14]$. However, most of researches on sleep quality during public health emergencies in China focus on patients or health care workers. Pinto and Zhou found that patients and medical staff struggled with COVID-19 every day and generally had poor sleep quality $[11,12]$. Nowadays, there are few studies on sleep quality and its influencing factors in the ordinary population. This study 
revealed that $41.1 \%$ of the general public suffered from poor sleep quality during the normalization of COVID-19 pandemic prevention and control. A survey of front-line health care workers in China showed that the prevalence of poor sleep quality was $61.6 \%$, which is far higher than $41.1 \%$ in our study [37]. The front-line health care workers faced with huge stress at work and prolonged working hours, which might lead to poorer sleep quality [38]. Another study reported that the prevalence of poor sleep quality at the beginning of the COVID-19 pandemic in China was 39.4\%, which was similar to our study (41.1\%) [39]. These results indicated that as the duration of the COVID-19 pandemic has extended, the impact of the pandemic on the public sleep quality has not diminished. During the normalization of COVID-19 pandemic prevention and control in China, people still felt threatened by the resurgence of the pandemic and worried about the uncertainty of the future. All of these can affect people's sleep quality. Therefore, the sleep quality issue needs extensive and sustained attention during the normalization of COVID-19 pandemic prevention and control in China. People with poor sleep quality should be identified and managed in time, and a series of effective measures should be taken to improve sleep quality.

Consistent with our hypothesis, results from regression analysis and forest plot supported the significant association between the stress of COVID-19 pandemic and sleep quality. During the COVID-19 pandemic, individuals with higher perceived stress tend to have lower sleep quality. However, during the COVID-19 pandemic, there are many different sources of stress, and the reasons for this stress are also different. Firstly, during the COVID-19 pandemic, Hetkamp believed that the stress caused by the public's fear of viral infections will lead to a decline in sleep quality [40]. Stress is a mediator between COVID-19 virus infection risk and poor sleep quality. The spread of highly transmissible and highly pathogenic viruses in the population caused panic, which increased individual stress, and ultimately resulted in poor sleep quality $[1,41]$. Moreover, reduced income, unemployment, and other financial stress also contribute to a decline in sleep quality $[4,42]$. During the COVID-19 pandemic, insomnia symptoms became more severe due to economic stress $[4,42]$. Next, the fear that foreigners or imported food may spread the virus will also cause stress on the domestic public $[18,19]$. This stress is related to the poor control of outbreaks and the continued pandemic of mutant viruses [36]. What's more, when people feel various stress caused by the COVID-19 pandemic, someone will experience compulsive checking, reassurance seeking behavior, and even more serious traumatic stress symptoms [20,43,44]. Sleep quality is often greatly affected when faced with the above-mentioned stress. Therefore, it is necessary to focus on the groups most affected by stress in the COVID-19 pandemic, and take timely psychological interventions to protect vulnerable groups with the goal of early detection.

There was also a positive association between worries and sleep quality during the COVID-19 pandemic. The higher the level of worries, the higher the incidence of insomnia [28]. Interestingly, our study highlights the worries about the future during the COVID-19 pandemic, which is similar to previous studies. Song's research showed that worries about future job losses and job changes can affect sleep quality [45]. People will lose their source of income and have no sense of security if unemployed, which may lead to anxiety and depression, and ultimately lead to their poor sleep quality [45]. Moreover, Tasso found that students' worries about future academic performance were also linked to sleep disorders [46]. The shift to online learning and the lack of physical activity at home may be reasons for students' 
worries. In summary, researchers need to take further steps to address the worries of the general public in order to improve their sleep quality.

Specifically, resilience appeared to be a protective factor for sleep quality. When suffering from stress and trauma, a strong level of resilience helps to improve the quality of sleep [30]. A large longitudinal study of pregnant women in Finland showed that higher levels of resilience predicted better sleep quality [47]. Similarly, during the COVID-19 pandemic, the active communication and resilience intervention helped health care workers quickly recover from COVID-19 and sleep quality improved [32]. However, lack of resilience can exacerbate psychological problems such as loneliness, stress and worries during the COVID-19 pandemic, which can increase symptoms of severe insomnia [27]. Therefore, resilience intervention should be paid attention to help improve the sleep quality of individuals who affected by COVID-19 pandemic.

The current study also found that related factors affecting sleep quality included chronic diseases, medical visits, occupation and negative life events. First of all, in terms of population health condition, patients with chronic diseases or patients with frequent medical visits during the COVID-19 outbreak had poorer sleep quality than the general population. This conclusion is similar to that of a study in Brazil [48]. He believed that people with chronic diseases were $36 \%$ more likely to have sleep disorders than people without them, and that people with diabetes had the highest rates of sleep problems [48]. Similarly, Courtney found that some patients with poor health and frequent medical treatment are at higher risk of sleep disorders [49]. This may be because people with poor health conditions can't meet their medical needs during the COVID-19 pandemic. Furthermore, the unmet needs may be related to local movement restrictions, inconvenient routine screening, medical treatment, and high economic costs during the COVID-19 pandemic [50]. Secondly, in terms of occupation, previous research has shown that individuals unemployed and retired have poorer sleep quality than employed population and college students. This could be because the unemployed had less disposable income, and worries about the economy and the future of their lives eventually lead to poorer sleep quality $[45,51]$. Retirees who typically spend more time at home, experience more daytime sleepiness, leading to poorer sleep quality at night [52]. Thirdly, similar to previous studies, individuals who experienced negative life events (divorce, death of relatives, lower income, job loss, etc.) tended to have poorer sleep quality $[42,53]$. That because negative life events experienced by individuals can cause psychiatric symptoms such as anxiety and stress, which can have a greater impact on sleep quality. This requires identifying individuals who have experienced negative life events, and preventing more severe sleep disorders.

\section{Limitations}

The results of this study should be considered with several potential limitations. Firstly, generalization of the results of this study to the whole population is limited by the convenience sampling method used to survey population information. In the present study, the average educational level of our participants would be higher than the average level of general population in China. Secondly, this study is a cross- 
sectional study, and the causal relationship between the study variables needs to be further confirmed by a cohort study or an experimental study.

\section{Conclusions}

During the normalization of COVID-19 pandemic prevention and control, the general public in China had poor sleep quality. Stress from all aspects of the pandemic, worries about the pandemic can lead to poor sleep quality and sleep problems. Resilience is a protective factor of sleep quality, and individuals with higher resilience have better sleep quality. On the one hand, psychological intervention should be carried out to improve the quality of sleep for those who are stressed and worried during the COVID-19 pandemic. On the other hand, people should be encouraged to receive resilience training and have a stronger mind in the face of difficulties, so as to achieve the goal of improving sleep quality. In addition, more efforts should be made to help people at risk of poor sleep quality, such as those with chronic diseases, high medical needs and negative life events, to help them regain confidence and improve their sleep quality.

\section{Declarations}

\section{Acknowledgments}

The authors wish to thank all the investigators who helped to conduct this survey and thank all the participants who gave their time to participate in this survey.

\section{Funding}

This work was financially supported by China Medical Board-Open Competition program (15-229). However, the sponsor did not participant in the preparation of the article.

\section{Ethics approval and consent to participate}

Ethics certification is awarded by the Ethics Committee of China Medical University. All participants voluntarily agreed to participate in the study.

\section{Competing interests}

The authors declare that they have no competing interests

\section{Authors' contributions}

The first author Ma mainly designed and wrote manuscripts for the research. Liu and Zhao mainly designed questionnaires and carried out the investigation process. Long and Diao mainly analyze data, and Feng and Wu mainly collect references and analyze data again. Corresponding author Wang supervised the entire research process, found problems and revised the original manuscript.

\section{Consent for publication}


Not applicable

\section{Availability of data and materials}

The datasets used and/or analysed during the current study are available from the corresponding author on reasonable request.

\section{References}

1. Shereen, M.A., et al., COVID-19 infection: Origin, transmission, and characteristics of human coronaviruses. J Adv Res, 2020. 24: p. 91-98.

2. (WHO), T.W.H.O. The World Health Organization (WHO) Has Officially Named the Disease Caused by the Novel Coronavirus as COVID-19. 2020 [cited 202011 February].

3. China, N.H.C.o.t.P.s.R.o. Real-time big data of the pandemic of COVID-19. 20217 April 2021.

4. Pak, A., et al., Economic Consequences of the COVID-19 Outbreak: the Need for Epidemic Preparedness. Frontiers in Public Health, 2020. 8.

5. Wang, Y., et al., The impact of quarantine on mental health status among general population in China during the COVID-19 pandemic. Mol Psychiatry, 2021.

6. Zhang, Y. and Z.F. Ma, Impact of the COVID-19 Pandemic on Mental Health and Quality of Life among Local Residents in Liaoning Province, China: A Cross-Sectional Study. Int J Environ Res Public Health, 2020. 17(7).

7. Liu, X., et al., Psychological status and behavior changes of the public during the COVID-19 epidemic in China. Infect Dis Poverty, 2020. 9(1): p. 58.

8. Xie, J., et al., Depressive Symptoms, Sleep Quality and Diet During the 2019 Novel Coronavirus Epidemic in China: A Survey of Medical Students. Front Public Health, 2020. 8: p. 588578.

9. Targa, A.D.S., et al., Decrease in sleep quality during COVID-19 outbreak. Sleep Breath, 2021. 25(2): p. 1055-1061.

10. Raniti, M.B., et al., Sleep Duration and Sleep Quality: Associations With Depressive Symptoms Across Adolescence. Behav Sleep Med, 2017. 15(3): p. 198-215.

11. Pinto, J., et al., Sleep quality in times of Covid-19 pandemic. Sleep Medicine, 2020. 74: p. 81-85.

12. Zhou, Y., et al., Prevalence and Demographic Correlates of Poor Sleep Quality Among Frontline Health Professionals in Liaoning Province, China During the COVID-19 Outbreak. Front Psychiatry, 2020. 11: p. 520 .

13. Stanton, R., et al., Depression, Anxiety and Stress during COVID-19: Associations with Changes in Physical Activity, Sleep, Tobacco and Alcohol Use in Australian Adults. Int J Environ Res Public Health, 2020. 17(11).

14. Ji Yanbin, W.Z., Chen Wenhao, Li Suxia, Xie Wen, Public sleep quality and related factors during COVID-19 epidemic period. Chinese Mental Health Journal, 2020. 34: p. 795-799. 
15. Xu, Y., et al., Parental psychological distress and attitudes towards COVID-19 vaccination: A crosssectional survey in Shenzhen, China. Journal of Affective Disorders, 2021. 292: p. 552-558.

16. Taylor, S., et al., Development and initial validation of the COVID Stress Scales. Journal of Anxiety Disorders, 2020. 72.

17. Dennis, D., C. Radnitz, and M.G. Wheaton, A Perfect Storm? Health Anxiety, Contamination Fears, and COVID-19: Lessons Learned from Past Pandemics and Current Challenges. Int J Cogn Ther, 2021: $\mathrm{p}$. $1-17$.

18. Chi, Y., et al., The Long-Term Presence of SARS-CoV-2 on Cold-Chain Food Packaging Surfaces Indicates a New COVID-19 Winter Outbreak: A Mini Review. Front Public Health, 2021. 9: p. 650493.

19. Zhu, J., et al., Challenges Caused by Imported Cases Abroad for the Prevention and Control of COVID19 in China. Frontiers in Medicine, 2021. 8.

20. Medina Fernandez, I.A., et al., Fear, Stress, and Knowledge regarding COVID-19 in Nursing Students and Recent Graduates in Mexico. Invest Educ Enferm, 2021. 39(1).

21. Scarpelli, S., et al., Pandemic nightmares: Effects on dream activity of the COVID-19 lockdown in Italy. J Sleep Res, 2021: p. e13300.

22. Liu, C., et al., The Combined Impact of Gender and Age on Post-traumatic Stress Symptoms, Depression, and Insomnia During COVID-19 Outbreak in China. Front Public Health, 2020. 8: p. 620023.

23. Fu, W., et al., Psychological health, sleep quality, and coping styles to stress facing the COVID-19 in Wuhan, China. Transl Psychiatry, 2020. 10(1): p. 225.

24. Tousignant, O.H., et al., Effects of Rumination and Worry on Sleep. Behav Ther, 2019. 50(3): p. 558570.

25. Wang, C., et al., Immediate Psychological Responses and Associated Factors during the Initial Stage of the 2019 Coronavirus Disease (COVID-19) Epidemic among the General Population in China. Int J Environ Res Public Health, 2020. 17(5).

26. Zysberg, L. and A. Zisberg, Days of worry: Emotional intelligence and social support mediate worry in the COVID-19 pandemic. Journal of Health Psychology, 2020.

27. Grossman, E.S., et al., COVID-19 related loneliness and sleep problems in older adults: Worries and resilience as potential moderators. Pers Individ Dif, 2021. 168: p. 110371.

28. Scotta, A.V., M.V. Cortez, and A.R. Miranda, Insomnia is associated with worry, cognitive avoidance and low academic engagement in Argentinian university students during the COVID-19 social isolation. Psychol Health Med, 2020: p. 1-16.

29. Friborg, O., et al., A new rating scale for adult resilience: what are the central protective resources behind healthy adjustment? Int J Methods Psychiatr Res, 2003. 12(2): p. 65-76.

30. Cai, Y., J. Wang, and L. Hou, Resilience Improves the Sleep Quality in Disabled Elders: The Role of Perceived Stress. Frontiers in Psychology, 2021. 12. 
31. Anstiss, T., Motivational interviewing in primary care. J Clin Psychol Med Settings, 2009. 16(1): p. 8793.

32. Huffman, E.M., et al., How resilient is your team? Exploring healthcare providers' well-being during the COVID-19 pandemic. Am J Surg, 2021. 221(2): p. 277-284.

33. Guo, S., et al., Structural Validity of the Pittsburgh Sleep Quality Index in Chinese Undergraduate Students. Frontiers in Psychology, 2016. 07.

34. Zhu, B., et al., Adaptation of the Pittsburgh Sleep Quality Index in Chinese adults with type 2 diabetes. Journal of the Chinese Medical Association, 2018. 81(3): p. 242-247.

35. Leal-Costa, C., et al., Psychometric properties of the Chinese version of the Resilience Scale (RS-14): Preliminary results. Plos One, 2020. 15(10).

36. Matta, S., et al., Covid-19 vaccines and new mutant strains impacting the pandemic. Indian J Tuberc, 2021. 68(2): p. 171-173.

37. Wang, W., et al., Sleep Disturbance and Psychological Profiles of Medical Staff and Non-Medical Staff During the Early Outbreak of COVID-19 in Hubei Province, China. Frontiers in Psychiatry, 2020. 11.

38. Tu, Z.-h., J.-w. He, and N. Zhou, Sleep quality and mood symptoms in conscripted frontline nurse in Wuhan, China during COVID-19 outbreak. Medicine, 2020. 99(26).

39. Yu, B.Y.-M., et al., Prevalence of sleep disturbances during COVID-19 outbreak in an urban Chinese population: a cross-sectional study. Sleep Medicine, 2020. 74: p. 18-24.

40. Hetkamp, M., et al., Sleep disturbances, fear, and generalized anxiety during the COVID-19 shut down phase in Germany: relation to infection rates, deaths, and German stock index DAX. Sleep Med, 2020. 75: p. 350-353.

41. Siddique, R.F., O. Ahmed, and K.N. Hossain, Relationship between the fear of COVID-19 disease and sleep quality: the mediating role of stress. Heliyon, 2021. 7(5): p. e07033.

42. Peltz, J.S., J.S. Daks, and R.D. Rogge, Mediators of the association between COVID-19-related stressors and parents' psychological flexibility and inflexibility: The roles of perceived sleep quality and energy. J Contextual Behav Sci, 2020. 17: p. 168-176.

43. Khosravani, V., et al., The Persian COVID stress scales (Persian-CSS) and COVID-19-related stress reactions in patients with obsessive-compulsive and anxiety disorders. Journal of ObsessiveCompulsive and Related Disorders, 2021. 28.

44. Hyun, S., et al., Psychological correlates of poor sleep quality among U.S. young adults during the COVID-19 pandemic. Sleep Medicine, 2021. 78: p. 51-56.

45. Song, L., et al., Mental Health and Work Attitudes among People Resuming Work during the COVID-19 Pandemic: A Cross-Sectional Study in China. International Journal of Environmental Research and Public Health, 2020. 17(14).

46. Tasso, A.F., N. Hisli Sahin, and G.J. San Roman, COVID-19 disruption on college students: Academic and socioemotional implications. Psychological Trauma: Theory, Research, Practice, and Policy, 
2021. 13(1): p. 9-15.

47. van der Zwan, J.E., et al., Longitudinal associations between sleep and anxiety during pregnancy, and the moderating effect of resilience, using parallel process latent growth curve models. Sleep Med, 2017. 40: p. 63-68.

48. Lima, M.G., et al., Effect of chronic non-communicable diseases (CNCDs) on the sleep of Brazilians during the COVID-19 pandemic. Sleep Med, 2021.

49. Polenick, C.A., N.R. Daniel, and E.A. Perbix, Factors Associated With Sleep Disturbances Related to the COVID-19 Pandemic Among Older Adults With Chronic Conditions. Am J Geriatr Psychiatry, 2021.

50. Wu, T., et al., Prevalence of mental health problems during the COVID-19 pandemic: A systematic review and meta-analysis. Journal of Affective Disorders, 2021. 281: p. 91-98.

51. Bartoszek, A., et al., Mental Well-Being (Depression, Loneliness, Insomnia, Daily Life Fatigue) during COVID-19 Related Home-Confinement-A Study from Poland. Int J Environ Res Public Health, 2020. 17(20).

52. Malakouti, S.K., et al., Sleep patterns, sleep disturbances and sleepiness in retired Iranian elders. International Journal of Geriatric Psychiatry, 2009. 24(11): p. 1201-1208.

53. Duran, S. and Ö. Erkin, Psychologic distress and sleep quality among adults in Turkey during the COVID-19 pandemic. Progress in Neuro-Psychopharmacology and Biological Psychiatry, 2021. 107.

\section{Figures}




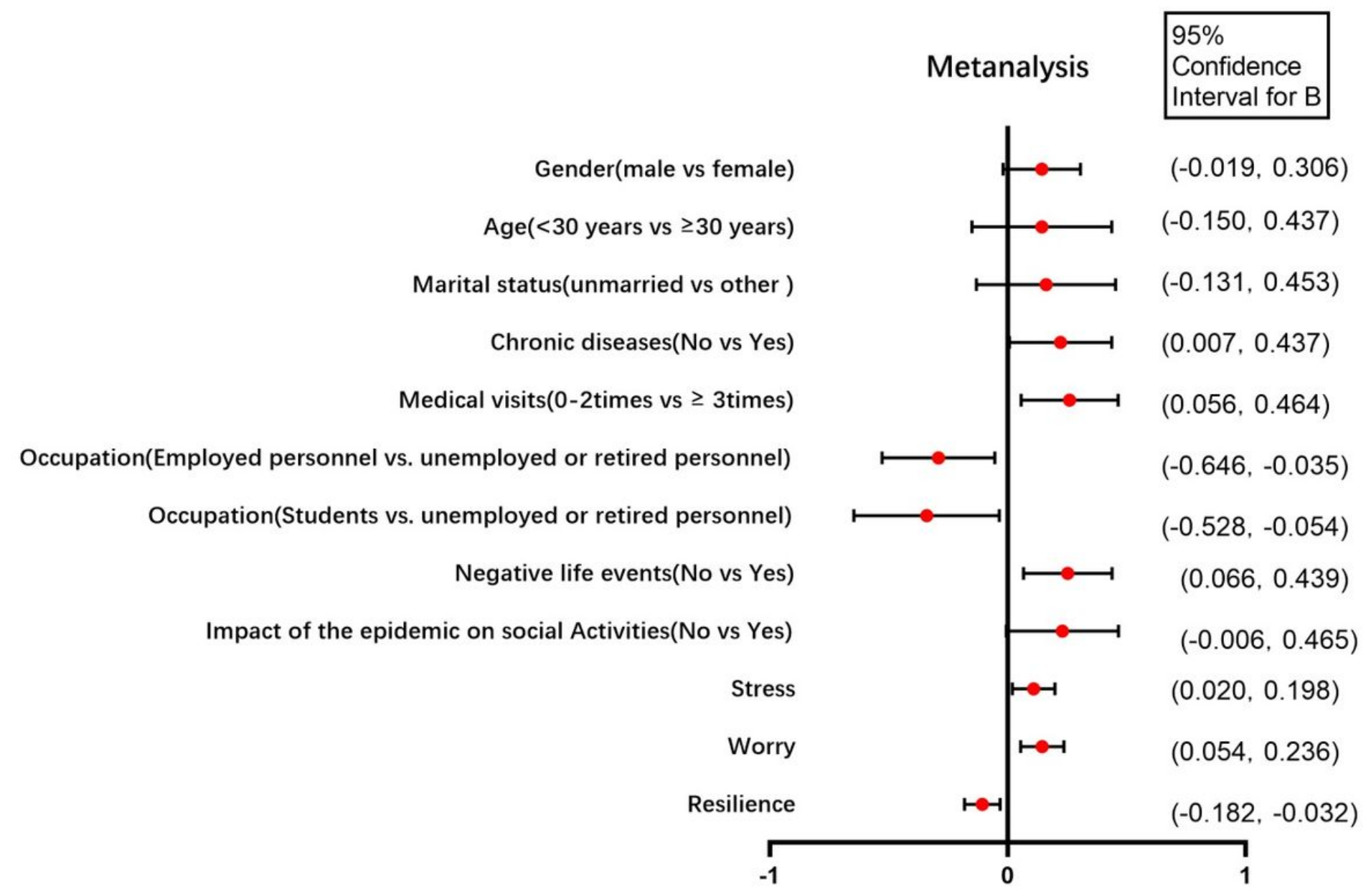

\section{Figure 1}

Forest plot of the related factors of sleep quality (hierarchical regression analysis). 